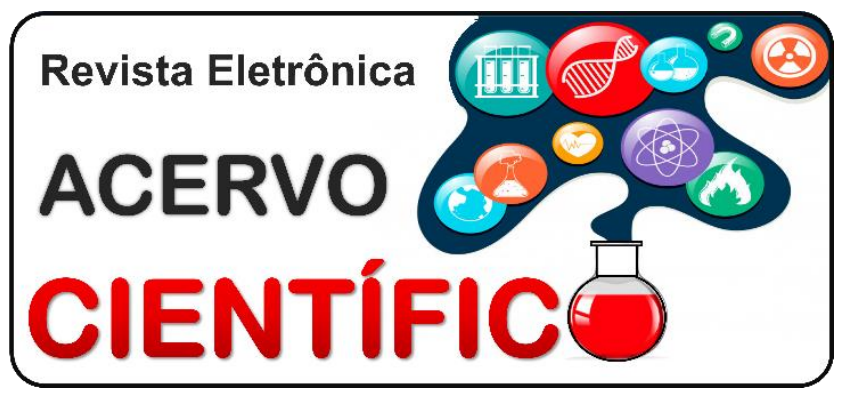

ARTIGO ORIGINAL

Recebido em: 7/2020

Aceito em: $8 / 2020$

Publicado em: 9/2020

\title{
Análise da mortalidade hospitalar por insuficiência cardíaca no estado de Minas Gerais, Brasil
}

\author{
Analysis of hospital mortality for heart insufficiency in the state of Minas Gerais, Brazil \\ Análisis de la mortalidad hospitalaria por insuficiencia del corazón en el estado de Minas \\ Gerais, Brasil
}

Henrique Guimarães Vasconcelos ${ }^{1 *}$, Sílvio Henrique da Silva Vaz ${ }^{1}$, Luciana Ferreira Rodrigues Prado $^{1}$, Larissa Caroline Rezende ${ }^{1}$, Anna Paula Braz Machado ${ }^{1}$, Davi Fernando Gomes Pereira ${ }^{1}$, João José Rios Maciel ${ }^{1}$, Jovelina de Oliveira Pereira ${ }^{1}$, Pedro Henrique de Freitas Abreu ${ }^{1}$, Vanessa Fernandes Andrade'.

\begin{abstract}
Resumo: Este trabalho buscou descrever a taxa de mortalidade hospitalar por insuficiência cardíaca (IC) no estado de Minas Gerais, de 2010 a 2019. Trata-se de estudo descritivo de cunho epidemiológico com base em informações coletadas no DATASUS. A mortalidade hospitalar por IC foi avaliada quanto à faixa etária, sexo e raça. O número de internações e de óbitos diminuiu, porém em proporções diferentes, de modo que a taxa de mortalidade anual foi maior ao fim do período. Os índices por faixa etária diminuíram em indivíduos de até 39 anos, com exceção do grupo entre 5-9 anos, no qual houve aumento significativo (22,07\%). A partir de 40 anos, os índices apresentaram elevação, mais importante acima dos 70 anos. Os números são discretamente maiores na população feminina quando comparada à masculina $(0,19 \%)$. As populações branca, amarela e indígena possuem maiores índices quando comparadas à parda e à preta. Observou-se, portanto, uma tendência de aumento da mortalidade hospitalar por IC em Minas Gerais a partir da quarta década de vida, além de disparidade entre as raças, sendo necessários estudos mais aprofundados para julgar fatores possivelmente associados. Conclui-se que a IC ainda é uma patologia complexa, de alta letalidade, sendo fundamental seu diagnóstico e manejo precoces.
\end{abstract}

Palavras-chave: Insuficiência cardíaca, Mortalidade hospitalar, Hospitalização.

\begin{abstract}
This study sought to describe the hospital mortality rate due to heart insufficiency (HF) in the state of Minas Gerais, from 2010 to 2019. This is a descriptive study of an epidemiological nature based on information collected in DATASUS. Hospital mortality due to HF was assessed in terms of age, sex and race. The number of hospitalizations and deaths decreased, but in different proportions, so that the annual mortality rate was higher at the end of the period. The indices by age group decreased in individuals up to 39 years old, except for the group between 5-9 years old, in which there was a significant increase $(22,07 \%)$. From the age of 40 , the indexes increased, most important above the age of 70 . The numbers are slightly higher in the female population when compared to the male population $(0,19 \%)$. The white, yellow and indigenous populations have higher rates when compared to brown and black. Therefore, in this study, there
\end{abstract}

1 Universidade de Itaúna (UIT), Itaúna-MG. *E-mail: guimaraes.henrique@yahoo.com 
was a tendency to increase hospital mortality due to HF in Minas Gerais from the fourth decade of life, in addition to the disparity between races, requiring further studies to judge possibly associated factors. It is concluded that HF is still a complex pathology, with high lethality, and its early diagnosis and management is essential.

Keywords: Heart failure, Hospital mortality, Hospitalization.

Resumen: Este estudio buscó describir la tasa de mortalidad hospitalaria por insuficiencia del corazón (IC) en el estado de Minas Gerais, de 2010 a 2019. Este es un estudio descriptivo de naturaleza epidemiológica basado en la información recopilada en DATASUS. La mortalidad hospitalaria por IC se evaluó en términos de edad, sexo y raza. El número de hospitalizaciones y muertes disminuyó, pero en diferentes proporciones, por lo que la tasa de mortalidad anual fue mayor al final del período. Los índices por grupo de edad disminuyeron en individuos de hasta 39 años, excepto en el grupo entre 5-9 años, en el que hubo un aumento significativo (22,07\%). A partir de los 40 años, los índices aumentaron, lo más importante por encima de los 70 años. Los números son ligeramente más altos en la población femenina en comparación con la población masculina $(0,19 \%)$. Las poblaciones blanca, amarilla e indígena tienen tasas más altas en comparación con el marrón y el negro. Por lo tanto, en este estudio, hubo una tendencia a aumentar la mortalidad hospitalaria debido a la insuficiencia cardíaca en Minas Gerais desde la cuarta década de la vida, además de la disparidad entre razas, lo que requiere más estudios para juzgar posibles factores asociados. Se concluye que la IC sigue siendo una patología compleja, con alta letalidad, y su diagnóstico y manejo tempranos son esenciales.

Palabras clave: Insuficiencia cardiaca, Mortalidad hospitalaria, Hospitalización.

\section{INTRODUÇÃO}

A Insuficiência Cardíaca (IC) é um mal crônico, oriunda de uma disfunção do miocárdio, e representa uma complicada síndrome clínica de aspecto sistêmico. Tal desordem resulta em uma perfusão sanguínea deficitária para as necessidades metabólicas teciduais (SOUZA TCTOA, et al., 2019). É importante ressaltar que a IC é o destino da maioria das doenças que afetam o coração, sendo, portanto, um dos grandes desafios clínicos da saúde na atualidade (POFFO MR, et al., 2017). Vale destacar que a doença também se classifica como um problema de grande peso na sociedade brasileira, visto que ocupa a segunda posição nas causas de morte por doenças cardiovasculares no país. Apresenta altos índices de hospitalizações em virtude das complicações clínicas, o que caracteriza a IC como um mal recorrente na saúde pública do Brasil há, aproximadamente, 20 anos (OSCALICES MIL, et al., 2020).

Segundo Polônia J, Gonçalves FR (2020), dentre a maquinaria fisiopatológica envolvida na IC, três aspectos têm maior destaque: a fibrose, a apoptose e o distúrbio ventricular. Estes estão vinculados a uma resposta inflamatória exagerada comumente presente em episódios de estresse do miocárdio, a título de exemplo, na hipertensão arterial sistêmica. A IC se manifesta, segundo termos clínicos, por um grupo de sinais que envolvem dilatação e hipertrofia do músculo cardíaco; modificação da capacidade de contração do coração, inotropismo e elevação da frequência cardíaca; ampliação da pré-carga e da pós-carga; e acúmulo renal de sódio e água. Em termos sintomatológicos, tais mudanças resultam em complicações respiratórias, turgidez, crepitações na ausculta, cansaço, estiramento abdominal, taquicardia, dentre outros. Diante disso, é perceptível que os afetados pela doença estão geralmente cerceados quanto à qualidade de vida, sobretudo no que diz respeito à prática de atividade física.

Os coeficientes de risco da IC incluem: senioridade avançada, sexo masculino, isquemia do músculo cardíaco, hipertensão, diabetes, dislipidemia, obesidade e tabaco. Surpreendentemente, a relevância aparente da doença se modifica com a região demográfica, com a isquemia do miocárdio a definir uma função hegemônica nos países ocidentais desenvolvidos (POLÔNIA J, GONÇALVES FR, 2020). A presença de outras comorbidades associadas à IC levam a uma piora no seu prognóstico. Em pacientes 
que apresentam IC aguda, a fibrilação atrial está presente em 20 a $30 \%$ destes. Já em pacientes ambulatoriais com IC, a insuficiência renal coexiste em $29,6 \%$ dos casos. Além disso, a hiponatremia, as múltiplas internações, os níveis elevados de peptídeo natriurético tipo B e a doença pulmonar associada são marcadores de mau prognóstico (POFFO MR, et al., 2017).

A IC atinge, aproximadamente, 23 milhões de pessoas e são diagnosticados 2 milhões de novos casos no mundo anualmente. Essa patologia, no Brasil, representa a maior causa de internações hospitalares em indivíduos acima de 60 anos (POFFO MR, et al., 2017). A cada ano, quase um milhão de internações são realizadas, indicando a elevada taxa de admissões hospitalares por esta síndrome, decorrendo, frequentemente, de fatores cardiovasculares, como as arritmias e as isquemias, e de fatores não cardiovasculares, como as infecções variadas, caracterizando a descompensação da IC (OSCALICES MIL, et al., 2020). O aumento de sobrevida em pacientes portadores de doenças cardiovasculares associado ao envelhecimento populacional contribui para manter a estabilidade ou, até mesmo, o aumento da prevalência desta patologia no Brasil e no mundo, ao contrário de algumas outras doenças cardiovasculares, em que se observa um declínio (WAGNER A, et al., 2017).

Sabe-se que a IC constitui cerca de $4 \%$ das internações gerais e atinge $31 \%$ das internações relacionadas às doenças cardiovasculares no Sistema Único de Saúde (SUS). Estima-se que 6,4 milhões de brasileiros apresentem a síndrome, sendo a cardiopatia isquêmica crônica, em associação à hipertensão arterial, sua mais importante etiologia (SOUZA TCTOA, et al., 2019). Na nação brasileira, a elevada taxa de morbimortalidade e os altos custos econômicos da IC têm como uma das bases as medicações introduzidas na fase hospitalar que, comumente, não seguem as diretrizes atuais (POFFO MR, et al., 2017). Considerando a alta taxa de morbimortalidade da IC, os escassos estudos epidemiológicos sobre a temática e a importância da epidemiologia para a discussão de propostas de prevenção em saúde pública, este estudo objetivou descrever a taxa de mortalidade hospitalar por IC no estado de Minas Gerais.

\section{MÉTODOS}

Trata-se de um estudo ecológico, descritivo de cunho epidemiológico realizado com base em dados da taxa de mortalidade por IC registrados no estado de Minas Gerais, que é constituído por 853 municípios e organizado em 10 macrorregiões de saúde (GOVERNO DO ESTADO DE MINAS GERAIS, 2010). De acordo com o Instituto Brasileiro de Geografia e Estatística (IBGE), o estado possui uma estimativa para o ano de 2020 de 21.292.666 habitantes, distribuídos em uma região de $586.521,121 \mathrm{~km}^{2}$.

A coleta de dados ocorreu no mês de março de 2020 por meio de registros do Departamento de Informática do Sistema Único de Saúde (DATASUS). Foram coletadas as taxas de mortalidade por IC correlacionadas ao sexo (masculino e feminino), faixa etária ( $\leq 1$ ano à $>80$ ) e raça/cor (branca, preta, parda, amarela e indígena), conforme a classificação do IBGE para categorizar a população brasileira, notificadas no período de 2010 a 2019.

Selecionou-se a taxa de mortalidade em concordância com o Capítulo IX - Doenças do Aparelho Circulatório (100-199), compreendendo as categorias de 150 a 1509 da $10^{\underline{a}}$ Classificação Internacional de Doenças e Problemas Relacionados à Saúde, 10ª Revisão (CID-10).

Os dados obtidos foram tabulados previamente pelo DATASUS com auxílio do programa Microsoft Office Excel (Microsoft@, 2019), analisados no mês de abril e maio de 2020 e apresentados por meio de estatística descritiva, com coeficiente de mortalidade obtido pela razão entre o número de óbitos e internações, multiplicada por 100 .

\section{RESULTADOS E DISCUSSÃO}

De acordo com a Tabela 1, observa-se um padrão de crescimento entre a proporção de óbitos e internações, inferindo aos dados estatísticos de morbidade e mortalidade por IC uma relevância dentro do grupo de doenças cardiovasculares, sobretudo no estado de Minas Gerais. 
Notabiliza-se que as taxas de mortalidade, que eram próximas de 8,5 na primeira metade do século XXI, subiram para valores aproximados de 9,8 . Além disso, mesmo com a diminuição do número de internação durante o período avaliado em $26,60 \%$, a queda do número de óbitos não seguiu a mesma proporção, sendo de apenas $15,22 \%$.

Na região sudeste, a cardiomiopatia chagásica representa ainda hoje, especialmente no Estado de Minas Gerais, a principal causa etiológica da IC, uma vez que a região está inserida na classificação de áreas endêmicas no país. Ainda que a Organização Pan-Americana da Saúde (OPAS) tenha certificado a erradicação da transmissão vetorial no Brasil, observam-se pequenos focos endêmicos no território brasileiro, mostrando a necessidade de estudos e controle dessa miocardiopatia especificamente (OPAS, 2019).

No que tange à estratificação da taxa de mortalidade por faixa etária e à comparação pelos anos de processamentos (Tabela 2), observou-se, entre os anos de 2010 e 2019, uma diminuição nas faixas etárias mais jovens, com exceção do grupo de crianças entre 5 e 9 anos de idade. Evidencia-se, no entanto, relativos aumentos dos índices de mortalidade nas faixas etárias mais velhas, com padrão maior de mortes com o avanço da idade. Infere-se ainda que, embora as taxas de mortalidade tenham se mostrado mais altas entre a população idosa e adulta no contexto geral, nas crianças menores de 1 ano e naquelas com idades entre 5 e 9 anos os índices de 8,65 e 8,7 foram superiores a todas as faixas adultas e idosas, com exceção dos indivíduos entre 70 e 79 e 80 anos e mais, cujos dados foram de 10,24 e 14,16.

Em relação ao intervalo de idade entre 5 e 9 anos supracitado, a explicação para este grupo ser destoante dos demais da infância e adolescência encontra-se no fato de tais crianças estarem inseridas em ambientes predisponentes à gênese da IC. Sabe-se, portanto, que esse número superior às faixas adultas e idosas está intimamente ligado à obesidade, à dislipidemia, à hipertensão arterial, à alteração dos níveis glicêmicos e ao sedentarismo, que são condições que têm sido observadas com elevada frequência nesse grupo etário e que constituem os principais fatores de risco da insuficiência cardíaca (PELLANDA LC, et al., 2010).

Além disso, observa-se que o período de 5 a 9 anos é marcado por uma grande dependência familiar, em que as crianças, por não possuírem um senso crítico formado em relação aos cuidados de saúde, acabam seguindo os comportamentos nocivos à saúde cardiovascular adotados pelos pais. Dessa forma, observa-se uma íntima relação dos números elevados de IC nessa faixa etária, mesmo que as faixas etárias vizinhas possuam um comportamento contrário devido às medidas protetivas e promocionais de saúde vigentes (FERREIRA JDF, et al., 2017).

De modo semelhante às crianças, é perceptível que a faixa etária compreendida entre 30 e 39 anos apresentou uma elevação na taxa de mortalidade por IC. Tal fato vincula-se aos índices aumentados de dislipidemia, que podem estar relacionados à genética dos indivíduos, ao estilo de vida e ao comportamento (SILVA VC, 2017). Sabe-se, ainda, que o indivíduo com níveis glicêmicos alterados possui o risco ampliado em 5 vezes para a ocorrência de IC em comparação com aquele que não possui diabetes mellitus (SOCIEDADE BRASILEIRA DE CARDIOLOGIA, 2019). O sobrepeso e a obesidade, por sua vez, estão associados ao estilo de vida das últimas décadas, caracterizado pelo grande consumo de alimentos industrializados de alto índice calórico e pelo baixo incentivo às práticas de atividade física recomendas pela Organização Mundial da Saúde (OMS, 2011).

Analisa-se que as faixas etárias entre 40 e 49, 50 e 59 e 60 e 69 anos, mesmo com maiores mortalidades naquelas de idades mais elevadas, seguiram um valor de proporção estável entre os anos de 2010 e 2019, diferentemente das faixas entre 20 e 29 e 30 e 39 anos, que mostraram relativo decréscimo e acréscimo, respectivamente. Ao observar as taxas de mortalidade nos indivíduos entre 70 e 79 e 80 anos ou mais, entre os anos já referidos, notabiliza-se respectivos aumentos significativos de $16,41 \%$ e $10,88 \%$. Nas crianças entre 5 e 9 anos, fugindo do padrão de diminuição de mortes entre os indivíduos abaixo dos 29 anos citado, observou-se aumento de mortalidade de $22,07 \%$. 
Tabela 1 - Internações, óbitos e taxa de mortalidade estratificada por ano de processamento. Minas Gerais, Brasil, $2010-2019$.

\begin{tabular}{ccccccccccccc}
\hline Variáveis & $\mathbf{2 0 1 0}$ & $\mathbf{2 0 1 1}$ & $\mathbf{2 0 1 2}$ & $\mathbf{2 0 1 3}$ & $\mathbf{2 0 1 4}$ & $\mathbf{2 0 1 5}$ & $\mathbf{2 0 1 6}$ & $\mathbf{2 0 1 7}$ & $\mathbf{2 0 1 8}$ & $\mathbf{2 0 1 9}$ & Total \\
\hline Internações & 38119 & 38995 & 37423 & 35307 & 33697 & 31795 & 31024 & 30861 & 30183 & 27976 & 337147 \\
Óbitos & 3238 & 3275 & 3157 & 2979 & 2867 & 2853 & 2896 & 2811 & 2890 & 2745 & 29892 \\
Taxa mortalidade & 8,49 & 8,4 & 8,44 & 8,44 & 8,51 & 8,97 & 9,33 & 9,11 & 9,57 & 9,81 & 8,87 \\
\hline
\end{tabular}

Fonte: Vasconcelos HG, et al., 2019. Baseado em DATASUS, 2019.

Tabela 2 - Taxa de mortalidade estratificada por faixa etária e ano de processamento. Minas Gerais, Brasil, $2010-2019$.

\begin{tabular}{cccccccccccc}
\hline Faixa Etária & $\mathbf{2 0 1 0}$ & $\mathbf{2 0 1 1}$ & $\mathbf{2 0 1 2}$ & $\mathbf{2 0 1 3}$ & $\mathbf{2 0 1 4}$ & $\mathbf{2 0 1 5}$ & $\mathbf{2 0 1 6}$ & $\mathbf{2 0 1 7}$ & $\mathbf{2 0 1 8}$ & $\mathbf{2 0 1 9}$ & Total \\
\hline Menor $\mathbf{1}$ ano & 12,63 & 9,57 & 7,55 & 9,47 & 5,52 & 5,88 & 6,29 & 6,13 & 10 & 8,65 & 8,26 \\
$\mathbf{1}$ a $\mathbf{4}$ anos & 4,4 & 3,23 & 5 & 9,09 & 7,35 & 3,41 & 7,25 & 5,77 & 8,16 & 4,08 & 5,74 \\
$\mathbf{5}$ a $\mathbf{9}$ anos & 6,78 & 4 & - & 5,56 & 5,41 & 2,13 & 6,67 & 10,53 & 4 & 8,7 & 5,04 \\
$\mathbf{1 0}$ a $\mathbf{1 4}$ anos & 5,26 & 1,67 & 2,38 & 9,38 & 14,29 & 4,88 & 2,5 & 6,67 & - & 4,35 & 5,18 \\
$\mathbf{1 5}$ a $\mathbf{1 9}$ anos & 5,94 & 8,24 & $\mathbf{4 , 6 7}$ & 13,64 & 6,25 & 10,67 & 5,13 & 2,94 & 11,9 & 5,26 & 7,46 \\
$\mathbf{2 0}$ a $\mathbf{2 9}$ anos & 7,37 & 5,01 & 5,79 & 5,08 & 6,25 & 7,28 & 6,47 & 4,64 & 5,26 & 5,49 & 5,87 \\
$\mathbf{3 0}$ a $\mathbf{3 9}$ anos & 5,47 & 5,2 & 5,46 & 5,48 & 5,88 & 5,92 & 6,74 & 5,82 & 6,82 & 4,84 & 5,71 \\
$\mathbf{4 0}$ a $\mathbf{4 9}$ anos & 5,84 & 5,39 & 5,29 & 5,39 & 6,06 & 6,09 & 5,91 & 5,59 & 5,67 & 6,88 & 5,8 \\
$\mathbf{5 0}$ a $\mathbf{5 9}$ anos & 6,19 & 5,96 & 6,04 & 6,52 & 5,78 & 6,3 & 6,36 & 6,28 & 6,94 & 6,95 & 6,3 \\
$\mathbf{6 0}$ a $\mathbf{6 9}$ anos & 7,49 & 7,22 & 7,06 & 7,09 & 6,5 & 7,32 & 7,31 & 7,45 & 7,83 & 7,71 & 7,3 \\
$\mathbf{7 0}$ a 79 anos & 8,56 & 8,75 & 8,46 & 8,8 & 8,66 & 8,89 & 9,51 & 9,16 & 9,56 & 10,24 & 9,01 \\
$\mathbf{8 0}$ anos e mais & 12,62 & 12,78 & 13,21 & 11,9 & 13,09 & 13,59 & 14,2 & 13,63 & 14,16 & 14,16 & 13,32 \\
\hline Total & 8,49 & 8,4 & 8,44 & 8,44 & 8,51 & 8,97 & 9,33 & 9,11 & 9,57 & 9,81 & 8,87 \\
\hline
\end{tabular}

Fonte: Vasconcelos HG, et al., 2019. Baseado em DATASUS, 2019. 
Associada à obesidade, a dislipidemia envolve o desequilíbrio patológico das taxas de lipídeos ou das lipoproteínas no sangue por questões genéticas (fator não modificável) e, também, por questões ambientais. Sabe-se que esta alteração orgânica tem maior relação com os hábitos alimentares e comportamentais do que com as condições genéticas do indivíduo. Entretanto, quando estes fatores estão presentes em associação, apresentam ação aditiva, intensificando o problema (SILVA VC, 2017).

Outro fator de risco importante é a hipertensão arterial, determinada pelo percentil da pressão arterial em relação à idade, sexo e estatura. Um indivíduo é considerado hipertenso quando apresenta valores iguais ou acima de $140 \mathrm{mmHg}$ para pressão arterial sistólica e $90 \mathrm{mmHg}$ para pressão arterial diastólica (SILVA VC, 2017). Para as crianças, a pressão arterial é considerada elevada quando os níveis pressóricos são maiores ou iguais ao percentil 90, sendo essenciais duas medidas para a confirmação do diagnóstico (SOCIEDADE BRASILEIRA DE CARDIOLOGIA, 2019). Ademais, as alterações dos níveis glicêmicos, que também possuem grande relevância na patogênese da IC, relacionam-se com a obesidade visceral, e o estado inflamatório vincula-se à resistência à insulina, o que caracteriza quadros de diabetes mellitus na infância secundários à obesidade e ao sedentarismo (SILVA VC, 2017).

Com as novas tecnologias sendo usadas de forma descontrolada, o abandono das atividades físicas tornou-se comum, tendo como consequência o sedentarismo. Os resultados da inatividade, como a falta de condicionamento cardiorrespiratório, podem ser fundamentais para o desenvolvimento da IC, além de serem agravantes dos fatores de risco (PELLANDA LC, et al., 2010).

Nos adultos é importante também considerar o tabagismo. As substâncias presentes no tabaco são extremamente prejudiciais para as pessoas que não possuem predisposição para doenças cardiovasculares, e são ainda mais danosas para os indivíduos que já apresentam predisposição para tais patologias, sendo um fator deletério nesta população (SOCIEDADE BRASILEIRA DE CARDIOLOGIA, 2019).

Nas pessoas acima de 70 anos, ocorreu um aumento significativo na mortalidade no período avaliado. Esses indivíduos, além de apresentarem os fatores de risco próprios da IC, são acometidos pelas comorbidades relacionadas ao envelhecimento, como obesidade visceral, a síndrome nefrótica, o hipotireoidismo e o uso de medicamentos (FERREIRA JDF, et al., 2017).

No que diz respeito à estratificação por cor/raça, infere-se que as populações branca, amarela e indígena, com 9,25, 10,5 e 12,08 mortes por 1.000 acometidos, possuíram maiores índices de mortalidade por IC no período analisado que as raças preta e parda, com 8,12 e 8,93, respectivamente (Gráfico 1).

Gráfico 1 - Taxa mortalidade por IC estratificada por cor/raça. Minas Gerias, Brasil, 2010-2019.

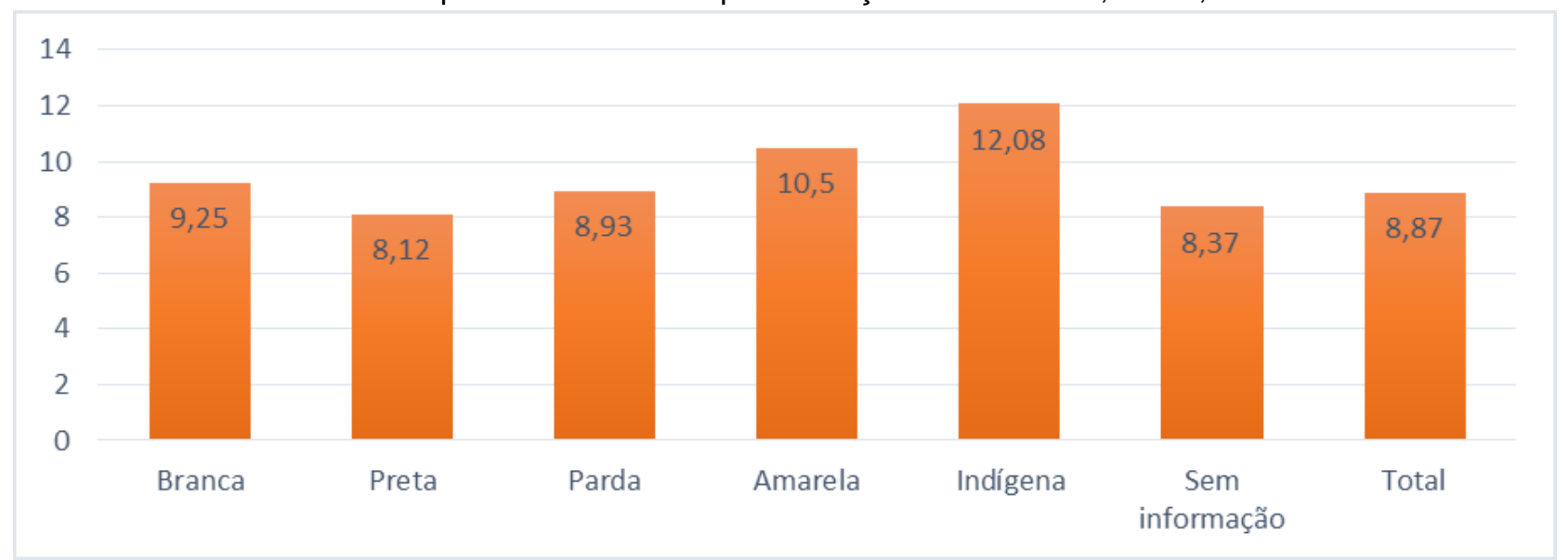

Fonte: Vasconcelos HG, et al., 2019. Baseado em DATASUS, 2019.

Estudos que avaliaram a mortalidade por IC em pacientes de diferentes etnias indicaram que as taxas de mortalidade entre negros são maiores do que em brancos usualmente (FERREIRA VEP, 2019; MILES JA, 
et al., 2019). Também foi demonstrado que pacientes de diferentes etnias tratados com os mesmos protocolos terapêuticos apresentaram desfechos distintos ao final dos ensaios clínicos, possivelmente devido às diferenças intrínsecas cardíacas. Por exemplo, pacientes negros com IC respondem menos ao tratamento com agentes moduladores de angiotensina, porém respondem melhor ao tratamento com nitratos-hidralazina (MILES JA, et al., 2019; WHITE M, et al., 2020).

Ao avaliar a taxa de mortalidade deste presente estudo, percebe-se que, percentualmente, a população negra morreu menos que a população branca entre 2010 e 2019 no estado de Minas Gerais. De acordo com Bui A, et al. (2011) e Lee W, et al. (2019), as possíveis disparidades encontradas na mortalidade entre as raças/etnias, como as encontradas na população indígena deste trabalho, necessitam de estudos mais aprofundados para julgar fatores que podem estar associados, como o acesso a medicação ou algum fator intrínseco dessa população.

A avaliação da população mineira de acordo com a cor/raça no quesito quantidade de internações por IC entre 2010 e 2019 evidencia que as populações indígena, amarela e preta, que correspondem à 10\% das internações, têm uma expressiva menor representatividade comparadas com brancos e pardos, que constituem $90 \%$ das internações. Este fato é consoante com as taxas de auto declaração étnicas brasileiras, pois $45,22 \%$ dos brasileiros se declaram brancos, $45,06 \%$ como pardos, $8,86 \%$ como pretos, $0,47 \%$ como amarelos e 0,38\% como indígenas de acordo com dados da Pesquisa Nacional por Amostra de Domicílios (PNAD 2015) (FERREIRA VEP, 2019).

A comparação das taxas de mortalidade por IC de acordo com sexo em Minas Gerais mostra que os números são discretamente maiores na população feminina, sendo que enquanto nos homens a IC mata 8,77 a cada 1.000 indivíduos, nas mulheres esse número é equivalente a 8,96 (Gráfico 2). A discreta maior taxa de mortalidade identificada no sexo feminio possivelmente se deve ao fato da longevidade dessa população ser mais elevada, havendo, portanto, mais comorbidades associadas à idade. No entanto, de acordo com Ho KKL, et al. (1993), no cenário nacional e no que diz respeito à distribuição por sexo, observou-se que $54,2 \%$ dos pacientes com IC eram do sexo masculino, dado similar ao dos estudos de Rassi S, et al. (2005) e Barretto ACP, et al. (1998).

Gráfico 2 - Taxa mortalidade estratificada por sexo. Minas Gerias, Brasil, 2010-2019.

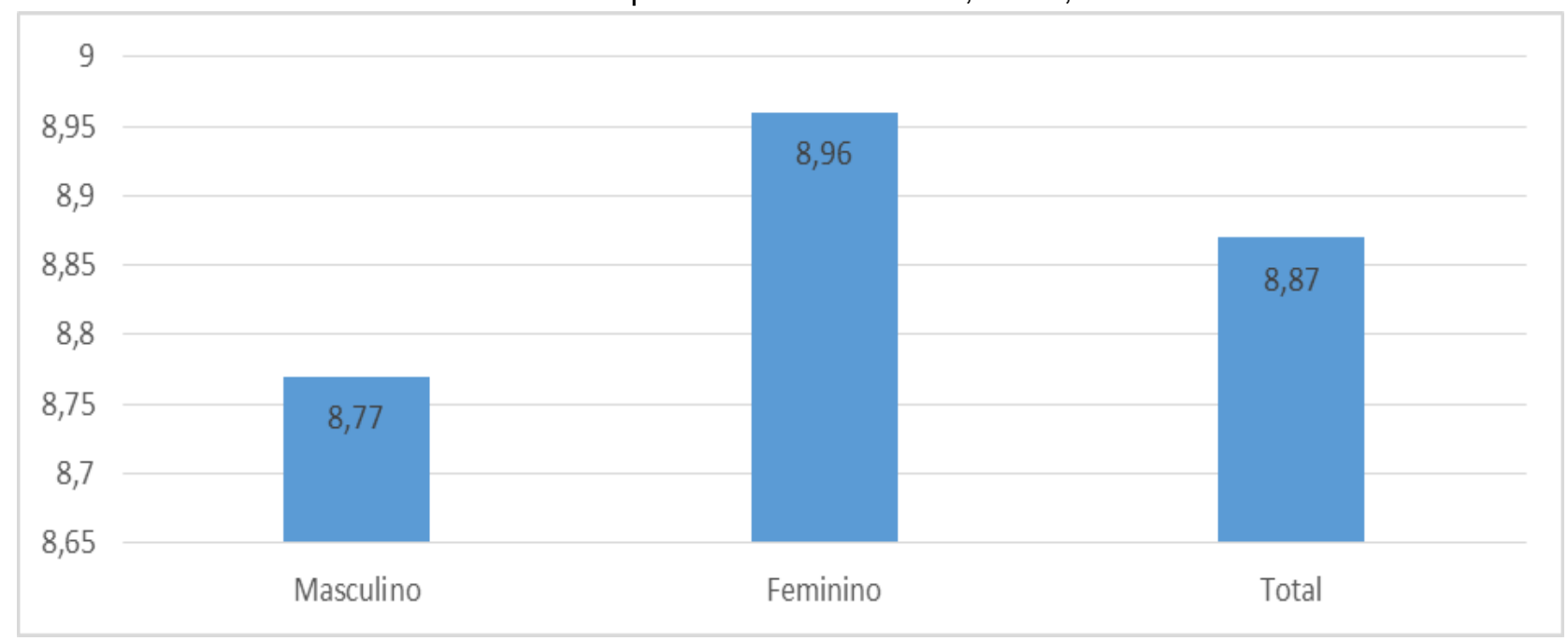

Fonte: Vasconcelos HG, et al., 2019. Baseado em DATASUS, 2019.

Em suma, fica evidente que proporcionar uma melhor condução dos casos de IC diagnosticados mostrase fundamental, objetivando tratamentos otimizados e mecanismos de atenção aos grupos de maior risco, como os de miocardiopatia hipertensiva e isquêmica. O intuito para tal centra-se na diminuição do número de internações, cirurgias e mortalidades e, de maneira geral, dos impactos socioeconômicos dessa síndrome (FIRMIDA CC, MESQUITA ET, 2001). 


\section{CONSIDERAÇÕES FINAIS}

A IC é uma enfermidade muito comum, de alta morbimortalidade, cujo diagnóstico, quando rápido e eficaz, é capaz de gerar melhores desfechos para os pacientes. Neste estudo, foi observado uma tendência de aumento na taxa de mortalidade hospitalar por IC a partir da quarta década de vida nos últimos dez anos em Minas Gerais. Faz-se necessário ressaltar que o número de internações e de óbitos diminuiu, provavelmente devido ao manejo otimizado e maior entendimento atual da doença, porém isso ocorreu em proporções diferentes, o que explica a taxa de mortalidade anual ser maior ao fim do período estudado. Com relação à discreta discrepância da taxa de mortalidades entre os sexos, ela possivelmente se deve ao fato de a longevidade na população feminina ser mais elevada, havendo mais comorbidades associadas à idade. As diferenças na taxa de mortalidade hospitalar entre as raças necessitam de mais estudos para julgar fatores possivelmente associados, como o acesso a medicação ou algum fator intrínseco dessas populações. Diante disto, medidas de prevenção de patologias do aparelho cardiovascular já devidamente comprovadas em estudos, como reeducação alimentar, controle de peso e prática de atividades físicas devem ser melhor difundidas entre a população, além da diminuição do tabagismo.

\section{REFERÊNCIAS}

1. BARRETTO ACP, et al. Insuficiência cardíaca em grande hospital terciário de São Paulo. Arq. Bras. Cardiol, 1998, 71(1): 15-20.

2. BARRETTO ACP, et al. Tratamento medicamentoso da insuficiência cardíaca em hospital terciário de São Paulo. Arq. Bras. Cardiol, 1997; 69(6): 375-379.

3. BUI A, et al. Epidemiology and risk profile of heart failure. Nat. Rev. Cardiol, 2011; 8: 30-41.

4. FERREIRA JDF, et al. Fatores de risco para doenças cardiovasculares em idosos. Rev. de Enfermagem UFPE On Line, 2017; 11(12): 4895-4905.

5. FERREIRA VEP. Insuficiência cardíaca na Paraíba: Análise epidemiológica de 2008 a 2018. Dissertação (TCC) Universidade Federal de Campina Grande, Paraíba, 2019, 57p.

6. FIRMIDA CC, MESQUITA ET. O paradoxo do tratamento da ICC com betabloqueadores: implicações para pacientes hipertensos. Rev. Bras. Hipertens, 2001; 8(4): 458-465.

7. FREIRE AKS. Panorama no Brasil das doenças cardiovasculares dos últimos quatorzes anos na perspectiva da promoção à saúde. Rev. Saúde e Desenvolvimento, 2017; 11(9): 1-24.

8. GOVERNO DO ESTADO DE MINAS GERAIS. 2010. In: Regiões de planejamento. Disponível em: https://www.mg.gov.br/sites/default/files/paginas/arquivos/2016/ligminas_10_2_02_regplan_listamunicipios.pdf. Acesso em: 19 abr. 2020.

9. HO KKL, et al. The epidemiology of heart failure: the Framingham study. J. Am. Coll. Cardiol, 1993; 22(A): 6-13.

10. IBGE. Instituto Brasileiro de Geografia e Estatística. 2020. In: Panorama. Disponível em: https://cidades.ibge.gov.br/brasil/mg/panorama. Acesso em: 19 abr. 2020.

11. LEE W, et al. Racial Disparities in Type of Heart Failure and Hospitalization. J Immigrant Minority Health, 2019; 21 : 98-104.

12. MILES JA, et al. Racial differences and mortality risk in patients with heart failure and hyponatremia. PLoS ONE, 2019; 14(6): e0218504.

13. NOGUEIRA RP, et al. Perfil epidemiológico, clínico e terapêutico da insuficiência cardíaca em hospital terciário. Arq. Bras. Cardiol, 2010; 95(3): 392-398.

14. ORGANIZAÇÃO MUNDIAL DE SAÚDE (OMS). Global recommendations on physical activity for health: 5-17 years old. Geneva: Organização Mundial de Saúde; 2011.

15. ORGANIZAÇÃO PAN-AMERICANA DA SAÚDE (OPAS). Guidelines for the diagnosis and treatment of Chagas disease. Washington: PAHO; 2019.

16. OSCALICES MIL, et al. Orientação de alta e acompanhamento telefônico na adesão terapêutica da insuficiência cardíaca: Ensaio clínico randomizado. Rev. Latino-Am. Enfermagem, 2019; 27: e3159.

17. PELLANDA LC, et al. Risco cardiovascular em crianças e adolescentes. Rev. Soc. de Cardiologia do Estado do RS, 2010; 20: 1-6.

18. POFFO MR, et al. Perfil dos Pacientes internados por insuficiência cardíaca em hospital terciário. Int. J. Cardiovasc. Sci, 2017; 30(3): 189-198.

19. POLÔNIA J, GONÇALVES FR. The historical evolution of knowledge of the involvement of neuro-hormonal systems in the pathophysiology and treatment of heart failure. Port. Soc. Cardiology, 2020; 1-13. 
20. RASSI S, et al. Sobrevida e fatores prognósticos na insuficiência cardíaca sistólica com início recente dos sintomas. Arq. Bras. Cardiol, 2005; 84(4): 309-313.

21. SILVA VC. Análise dos fatores de risco para doença cardiovasculares em crianças de 5 a 9 anos procedentes de escolas públicas da região central da cidade de Fortaleza, Ceará - Brasil. Dissertação (Doutorado) - Faculdade de Medicina da Universidade de São Paulo, 2017, 182p.

22. SOCIEDADE BRASILEIRA DE CARDIOLOGIA. Atualização da diretrizes de preservação Cardiovascular da Sociedade Brasileira de Cardiologia, 2019; 113(4): 1-105.

23. SOUZA TCTOA, et al. O difícil cotidiano dos pacientes com insuficiência cardíaca. Revista Online de Pesquisa, 2019; 11(5): 1340-1346.

24. WAJNER A, et al. Causas e preditores de mortalidade intra-hospitalar em pacientes que internam com ou por Insuficiência Cardíaca em Hospital Terciário no Brasil. Arq. Bras. Cardiol, 2017; 109(4): 321-330.

25. WHITE M, et al. Racial differences in mortality in patients with advanced systolic heart failure: Potential role of right ventricular ejection fraction. International Journal of Cardiology, 2020; 177(1): 255-260. 